\title{
A Comprasion of the Activation of Mirror Neurons Induced by Action Observation between Simple and Complex Hand Movement
}

\author{
Mi Young Lee ${ }^{1}$, Ju Sang Kim² \\ 'Department of Physical Therapy, College of Biomedical Science, Daegu Haany University, Gyeongsan; ${ }^{2}$ Department of Biomedical Science, Graduate \\ School, Daegu Haany University, Gyeongsan, Korea
}

Purpose: We compared the activation pattern of the mirror neurons (MN) between two types of hand movement according to action observation using functional MRI.

Methods: Twelve right-handed healthy subjects (5 male and 7 female, mean age $21.92 \pm 2.02$ years) participated in the experiment. During fMRI scanning, subjects underwent two different stimuli on the screen: 1) video clips showing repeated grasping and releasing of the ball via simple hand movement (SHM), and (2) video clips showing an actor performing a Purdue Pegboard test via complex hand movement (CHM). paired t-test in statistical parametric mapping (SPM) was used to compare the activation differences between the two types of hand movement.

Results: CHM as compared with the SHM produced a higher blood oxygen level dependent (BOLD) signal response in the right superior frontal gyrus, left inferior and superior parietal lobules, and lingual gyrus. However, no greater BOLD signal response was found by SHM compared with CHM (FWE corrected, $\mathrm{p}<0.05$ ).

Conclusion: Our findings provided that the activation patterns for observation of SHM and CHM are different. CHM also elicited boarder or stronger activations in the brain, including inferior parietal lobule called the MN region.

Keywords: Action observation, Mirror neuron, fMRI

\section{INTRODUCTION}

Mirror neurons (MNs) are activated when an individual performs an action or observes actions performed by others. ${ }^{1}$ In the 1990 s, NMs were first discovered; they denoted neuronal activity in the ventral premotor area (area F5) and inferior parietal lobule of a macaque monkey, both when the monkey executed a specific action and when they observed another monkey performing a similar action. ${ }^{2-4}$ Brain imaging and neurophysiological studies have revealed that MNs are activated in the human brain when observing actions performed by others. In the human brain, the area of homologous MNs is comprised of the inferior parietal lobule, superior temporal sulcus, and inferior frontal gyrus., ${ }^{1,5}$

In addition, MNs are associated with various human movements

Received May 16, 2019 Revised Jun 19, 2019

Accepted Jun 28, 2019

Corresponding author Ju Sang Kim

E-mail wntkd-c@hanmail.net involved with motor preparation and motion imitation. MNs also play an important role in the learning of motor patterns during action observation. ${ }^{6-8}$ Thus, various therapeutic interventions that can activate NMs have been applied, including action observation training and action execution with mirror visual feedback in the field of rehabilitation. ${ }^{9-11}$ In this regard, many previous studies have reported that action observation improves not only the function of upper limbs, but also the gait ability in stroke patients. ${ }^{12-17}$

MNs have gained popularity in research, which may lead to the discovery of new therapies in the field of rehabilitation. We suggest that visual information used for observation will be important for therapeutic effects. Therefore, it is necessary to verify whether the $\mathrm{MNs}$ reflect the features of the observed action. Therefore, we compared the activation pattern of MNs according to action observa-

Copylight (C)2019 The Korean Society of Physical Therapy

This is an Open Access article distribute under the terms of the Creative Commons Attribution Non-commercial License (Http:// creativecommons.org/license/by-nc/4.o.) which permits unrestricted non-commercial use, distribution, and reproduction in any medium, provided the original work is properly cited. 
tion based on the types of hand movement using functional MRI.

\section{METHODS}

\section{Subjects}

Twelve right-handed healthy subjects (5 males and 7 females) between the ages of 20 and 27 years (mean age $21.92 \pm 2.02$ years) with no history of neurological, physical, or psychiatric illness were enrolled for this experiment. The study was conducted in accordance with the Declaration of Helsinki. All subjects understood the purpose of the study and provided written, informed consent prior to participation. All subjects provided signed written informed consent forms.

\section{Functional MRI}

\section{1) Experimental paradigm}

All subjects were examined in supine position and were asked to not move during the experiment. An MRI compatible VisualSystem (NordicNeuroLab, Bergen, Norway) was used to present visual stimuli, and images projected on the screen were displayed through video goggles fixed in place with a head coil. The fMRI block design consisted of a stimulus condition (20 seconds) and a baseline condition (20 seconds). During fMRI scanning, subjects viewed two different stimuli on the screen: 1) video clips showing an actor performing repeated grasping and releasing of the ball via simple hand movement (SHM), and (2) video clips showing an actor performing a Purdue Pegboard test via complex hand movement (CHM). Purdue Pegboard test was performed as following; actor picks the pin, washer, and collar in the cup one by one and assembles them in the hole in the order of pin, washer, collar, and washer. The baseline blocks showed a fixation cross located in the center of a blank screen. Two alternative cycles were repeated five times and a run duration of one cycle was performed for 3 minutes and 20 seconds.
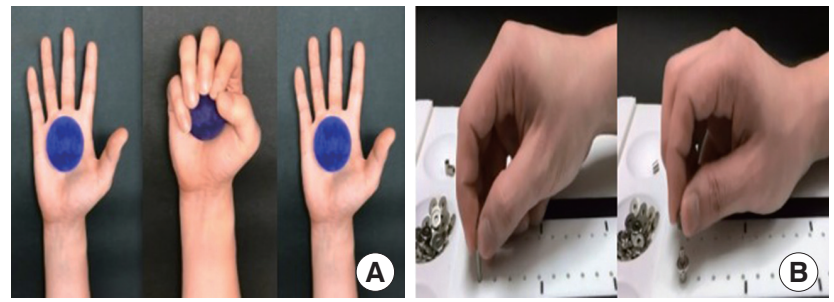

Figure 1. Type of observed action (A) simple hand movement (SHM) involving the repeated grasping and releasing of a ball. (B) Complex hand movement (CHM) involving performing a Purdue Pegboard test.
Sequence of the different stimuli was assigned randomly. The experimental block paradigm is shown in Figure 1.

\section{2) fMRI parameters}

The Magnetom Skyra 3T MRI system (Siemens, Erlangen, Germany) and the standard head coil were used to perform blood oxygenation level-dependent (BOLD) fMRI. BOLD-weighted Echo Planar Imaging $(\mathrm{EPI})$ parameters were as follows: repetition time $(\mathrm{TR})=2,000$ msec, echo time $(\mathrm{TE})=30 \mathrm{msec}$, field of view $(\mathrm{FOV})=210 \mathrm{~mm}$, flip angle $=90^{\circ}$, matrix size $=64 \times 64$, and slice thickness $=4 \mathrm{~mm}$. For anatomical reference images, 28 axial, $4 \mathrm{~mm}$ slice thick, T1-weighted, and spin echo images were obtained with a matrix size of $128 \times 128$, and an FOV of $210 \mathrm{~mm}$. Total images were acquired parallel to the bicommissure line of the anterior commissure-posterior commissure.

\section{3) fMRI data analysis}

An analysis of the fMRI data was performed using a statistical parametric mapping software (SPM 8, Welcome Department of Cognitive Neurology, London, UK), which was implemented in the MATLAB environment (The Mathworks, USA). All images were realigned and normalized, and then, smoothed spatially with a Gaussian kernel at a full width at half maximum (FWHM) of $8 \mathrm{~mm}$ to improve the signalto-noise ratio. The first level of analysis for each subject's contrast images was conducted to investigate the individual brain activation maps. The second level of analysis was performed using a random effect model with one-sample t-tests to examine the activation pattern of each stimulus conditions. Paired t-test was also used to compare the activation differences between the two types of hand movement. Activations were based on clusters larger than 10 contiguous voxels. Statistical significance was considered at an FWE (Family-wise error rate) corrected threshold of $\mathrm{p}<0.05$.

\section{RESULTS}

CHM produced a higher BOLD signal response in the right superior frontal gyrus, left inferior and superior parietal lobule, and lingual gyrus when compared with SHM. However, no greater BOLD signal response was found by SHM compared with CHM (FWE corrected, $\mathrm{p}<0.05$ )(Table 1)(Figure 2). 
Table 1. Brain activation difference between simple and complex hand movements

\begin{tabular}{|c|c|c|c|c|c|c|}
\hline \multirow{2}{*}{ Brain region } & \multirow{2}{*}{ Brodmann area } & \multicolumn{3}{|c|}{ Peak MNI coordinates } & \multirow{2}{*}{ Peak t-value } & \multirow{2}{*}{ No. of voxels } \\
\hline & & $x$ & $\mathrm{y}$ & z & & \\
\hline \multicolumn{7}{|c|}{ Complex movement $>$ Simple movement } \\
\hline Right superior frontal gyrus & 6 & 26 & 8 & 58 & 13.55 & 45 \\
\hline Left inferior parietal lobule & 40 & -40 & -28 & 38 & 12.15 & 22 \\
\hline Superior parietal lobule & 7 & -34 & -48 & 52 & 11.81 & 21 \\
\hline Lingual gyrus & 18 & -8 & -70 & -2 & -11.22 & 29 \\
\hline \multicolumn{7}{|c|}{ Simple movement $>$ Complex movement } \\
\hline NS & & & & & & \\
\hline
\end{tabular}

p-value is FWE corrected at $p<0.05$.

NS: non-significant.
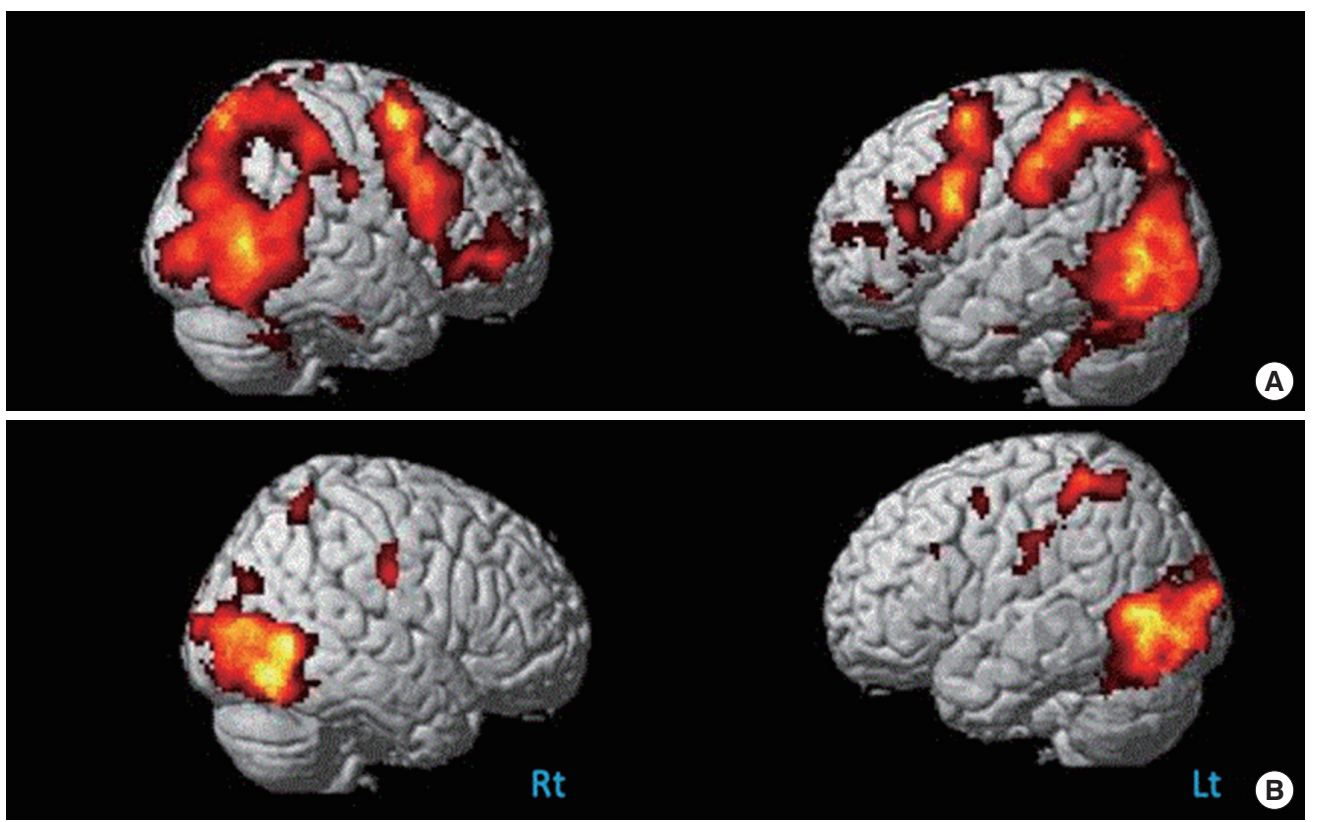

Figure 2. Results of the fMRI group analysis. Brain activations were shown during the observation for complex hand movement (A) and simple hand movement $(B)$ (uncorrected, $p<0.001$ ).

\section{DISCUSSION}

The present study investigated the activation pattern of MNs in accordance with the characteristics of observed actions. For that, we compared the activation of MNs while observing an action being performed between simple and complex hand movements. We found that $\mathrm{CHM}$ induced more significant activation in the right superior frontal gyrus, left inferior and superior parietal lobule, and lingual gyrus than SHM.

Research on MNs in humans showed consistent progress and development of brain imaging techniques, like the use of transcranial magnetic stimulation, fMRI, and other methods. ${ }^{6,18-22}$ Buccino et al. ${ }^{20}$ investigated the somatotopic organization of MNs. They showed that the activations in the premotor and parietal area corresponded to the type of movements used in the observed action. According to the results, when participants observe an action being performed with the mouth, arm/hand, and foot, the brain was activated in different parts of the frontal and parietal cortex. In detail, these activations showed a somatotopic organization with the activation shifting from the ventral to dorsal with respect to the observation of action in the mouth, hand, and foot. These findings have supported the assumption that MNs may reflect action perception.

The visual information obtained from observing an action is processed in the occipital lobe of the lingual gyrus and middle occipital gyrus. This visual information is then transferred to the inferior parietal lobule for specific kinesthetic coding. This information is pro- 
cessed in the inferior frontal gyrus, where the goal of the action is defined. ${ }^{1,18,21}$ Our results indicate that complex action observations showed stronger brain activations in visual information processing.

In addition, as a brain imaging study on the characteristics of movement, Park et al. ${ }^{23}$ compared brain activations between executed complex and simple movement using fMRI. They reported that stronger activation of ipsilateral hemisphere during execution of complex movement. As with brain activations for executed movement, our findings suggest that MNs might be also able to detect the complexity of observed actions.

In the current study, we compared the activation of MNs while observing an action between SHM and CHM. Our findings show that the activation patterns are different between SHM and CHM. CHM also elicited boarder or stronger activations of some brain areas, including the inferior parietal lobule called the MNs region. Therefore, we suggest that NMs may reflect the complexity of the observed actions. In the clinical field, clinical practices based on MNs activation may need to consider the contents of action observation presented. There are some limitations in this study. First, the sample size is small. Therefore, careful interpretation of the results may be necessary. Second, we only identified differences of brain activation without clinical data. So, we do not know functional changes related to brain activations. Third, because this study investigated the immediate brain activations during fMRI scanning, we did not demonstrate the brain activity for the long-term influence of action observation for promoting plasticity. These issues will be addressed in future studies.

\section{ACKNOWLEDGEMENTS}

This work was supported by the Basic Science Research Program through the National Research Foundation of Korea (NRF) funded by the Ministry of Education (No. 2017R1D1A1B03033985).

\section{REFERENCES}

1. Rizzolatti G, Craighero L. The mirror-neuron system. Annu Rev Neurosci. 2004;27:169-92.

2. Gallese V, Fadiga L, Fogassi L et al. Action recognition in the premotor cortex. Brain. 1996;119(Pt 2):593-609.

3. Rizzolatti G, Fadiga L, Gallese V et al. Premotor cortex and the recognition of motor actions. Brain Res Cogn Brain Res. 1996;3(2):131-41.

4. Rizzolatti G, Fadiga L, Matelli M et al. Localization of grasp representa- tions in humans by pet: 1. observation versus execution. Exp Brain Res. 1996;111(2):246-52.

5. Garrison KA, Winstein CJ, Aziz-Zadeh L. The mirror neuron system: a neural substrate for methods in stroke rehabilitation. Neurorehabil Neural Repair. 2010;24(5):404-12.

6. Buccino G, Vogt S, Ritzl A et al. Neural circuits underlying imitation learning of hand actions: an event-related fMRI study. Neuron. 2004; 42(2):323-34.

7. Fadiga L, Fogassi L, Pavesi G et al. Motor facilitation during action observation: a magnetic stimulation study. J Neurophysiol. 1995;73(6):2608-11.

8. Frenkel-Toledo S, Liebermann DG, Bentin S et al. Dysfunction of the human mirror neuron system in ideomotor apraxia: evidence from $\mathrm{mu}$ suppression. J Cogn Neurosci. 2016;28(6):775-91.

9. Altschuler EL, Wisdom SB, Stone L et al. Rehabilitation of hemiparesis after stroke with a mirror. Lancet. 1999;353(9169):2035-6.

10. Sarasso E, Gemma M, Agosta F et al. Action observation training to improve motor function recovery: a systematic review. Arch Physiother. 2015;5:14.

11. Kim JY, Park JW, Kim SY. The effects of functional electrical stimulation combined with action observation on sensorimotor cortex. J Kor Phys Ther. 2017;29(4):164-8.

12. Franceschini M, Ceravolo MG, Agosti M et al. Clinical relevance of action observation in upper-limb stroke rehabilitation: a possible role in recovery of functional dexterity. a randomized clinical trial. Neurorehabil Neural Repair. 2012;26(5):456-62.

13. Harmsen WJ, Bussmann JB, Selles RW et al. A mirror therapy-based action observation protocol to improve motor learning after stroke. Neurorehabil Neural Repair. 2014;29(6):509-16.

14. Park HR, Kim JM, Lee MK et al. Clinical feasibility of action observation training for walking function of patients with post-stroke hemiparesis: a randomized controlled trial. Clin Rehabil. 2014;28(8):794-803.

15. Kim JC, Lee HM. The effect of action observation training on sit to walk with chronic stroke patients. J Kor Phys Ther. 2015;27(6):413-8.

16. Song YH, Lee HM. The effect of treadmill training applied simultaneously with action observation on walking ability in chronic stroke patients. J Kor Phys Ther. 2016;28(3):176-82.

17. Kim HM, Song SM. Effect of action observation training with auditory feedback for gait function of stroke patients with hemiparesis. J Kor Phys Ther. 2017;29(5):246-54.

18. Arbib MA, Billard A, Iacoboni M et al. Synthetic brain imaging: grasping, mirror neurons and imitation. Neural Netw. 2000;13(8-9):975-97.

19. Aziz-Zadeh L, Koski L, Zaidel E et al. Lateralization of the human mirror neuron system. J Neurosci. 2006;26(11):2964-70.

20. Buccino G, Binkofski F, Fink GR et al. Action observation activates premotor and parietal areas in a somatotopic manner: a fMRI study. Eur J Neurosci. 2001;13(2):400-4.

21. Iacoboni M, Molnar-Szakacs I, Gallese V et al. Grasping the intentions of others with one's own mirror neuron system. PLoS Biol. 2005;3(3):e79.

22. Molnar-Szakacs I, Kaplan J, Greenfield PM et al. Observing complex action sequences: the role of the fronto-parietal mirror neuron system. Neuroimage. 2006;33(3):923-35.

23. Park JW, Kwon YH, Lee MY et al. Brain activation pattern according to exercise complexity: a functional MRI study. NeuroRehabilitation. 2008; 23(3):283-8. 